\title{
Analysis of air termination system of the lightning protection scheme for the Indian satellite launch pad
}

\author{
U. Kumar and N.T. Joseph
}

\begin{abstract}
Any satellite launch mission is a complex and expensive process and great care and precautions need to be taken for a safe and successful launch. A lightning stroke can be hazardous to the satellite launch system. Therefore extensive care has to be taken to protect the satellite launch pad against lightning. The paper aims at evaluating the efficacy of the aerial receptor system of the lightning protection scheme employed for protecting the Indian satellite launch pad. Significant upward leader activities are expected from tall objects during the approach of the descending leader and therefore any method that is employed for evaluating their attractive radii must take into account the different process involving and connected with the breakdown of air. Among such models the Rizk model, originally developed for isolated towers and conductors, was found to be very appropriate for the present work. To apply it to the present problem many modifications were necessary; all the simulations were carried out using this modified model. The charge simulation method has been used for the computation of the electric field. Simulations with inclined downward leaders indicated that the vertical leader descent represents the worst case scenario, and hence all the calculations were carried out with a vertical leader descent only. The maximum current in a bypass stroke was found to be $14 \mathrm{kA}$ and this occurs for a stroke descending vertically along a straight line directly above the launch vehicle. The total probability of bypass strokes was found to be in the range of one flash per 1095-510 years (for the value of $T d$ which is reported to be between $50-90$ thunderstorm-days per year). Similarly, the total number strokes to the protection system in an year was estimated to be less than two flashes per year. Further, some sensitivity analysis was also carried out to determine the dependency of bypass stroke on height of the protection system and total length of the descending leader. An attempt was also made to arrive at a rough estimate of maximum bypass current under positive lightning.
\end{abstract}

\section{Introduction}

Any satellite launch mission is a very complex and expensive process and therefore great care and precautions are to be taken for safe and successful launch. A lightning stroke can be hazardous to the satellite launch system. The large magnitudes of rate of rise and the peak current amplitudes associated with the lightning discharges can lead to very adverse electromagnetic, electrothermal and electromechanical effects.

This problem is further aggravated when the launch site is located at a place having a high isokeraunic level, as in the case of Indian satellite launch pad (reported to be in the range of 50-90 thunderstorm-days per year). Considering these, a lightning protection system (LPS) has been built for protecting the launch pad against direct strokes [1,2]. The three major components of the protection system [1] are:

- Air termination system: Consists of towers with masts and interconnecting shield wires. Three $120 \mathrm{~m}$ tall towers are situated at the vertices of an equilateral triangle of side approx. $180 \mathrm{~m}$. A $10 \mathrm{~m}$ mast with a number of cross-arms is



Fig. 1 Schematic of protection system along with launch vehicle (LV) and umbilical tower (UT)

the air termination system along with the umbilical tower (UT) and the launch vehicle (LV).

- Down conductor system: Towers themselves constitute a good down conductor system.

- Earth termination system: Radial and circular counterpoises with driven rods [1] 
In the design adopted for LPS, both air termination and down conductor systems are physically isolated from the launch pad. As a consequence, 100\% protection against direct strokes is not possible and a few strokes with current less than the critical value bypass the protection system [1]. These strokes for which shielding failure can occur are termed bypass strokes and their respective currents are called bypass currents. Two considerations have mainly governed the geometrical design of the air termination system. First, all strokes with current magnitudes higher than the specified value have to be intercepted by the LPS. Secondly, the induced potentials in the air termination/ down conductor systems during a stroke interception should not cause a flashover to the launch pad. For the initial design of the air termination system, the well-known rolling sphere method was employed for the computation of bypass currents and a simulation using the distributed circuit representation of the LPS was employed for the estimation of poststroke potentials [1]. The initial design was constrained by time limitations and therefore a detailed analysis was taken up. This work reports the results of the detailed investigations carried out for the bypass strokes and strokes to LPS.

As the stepped leader approaches ground the field induced on the grounded structures can become sufficient to generate upward leaders. In the literature on lightning, the terms upward leader and streamer have often been used interchangeably [3]. The final bridging of the descending leader and the upward leaders/streamers completes the stroke termination. Therefore for an accurate evaluation of the protective action of any LPS it is necessary that the procedure adopted must have representations for the different stages in the bridging process. This consideration becomes more important specifically for tall and slender objects, wherein considerable upward leader activities are expected before the final bridging occurs.

Due to the complexity of the associated phenomena many simplified methods have been developed. For simple geometries, as with high-voltage transmission lines, the design of protection system using the electro-geometric model (EGM) [4] was found to be satisfactory. The basis for this method is the concept of striking distance $r_{s}$ (in metres) given by the formula

$$
r_{s}=a l^{c}
$$

where $a$ ranges between $6-10$, the exponent $c$ has a value between $0.6-0.8$ and $\mathrm{Z}$ is the prospective stroke current peak in kA. For more complex structures the rolling sphere method was developed with radius of the sphere determined from (1) or a similar equation [5]. For shorter (height 20$30 \mathrm{~m})$ as well as flat-topped structures, significant upward leaders are not expected and as a result final bridging occurs mostly through streamers. These two methods wherein there is no explicit reference to the field intensification at the structure top as well as the upward leader activity, were found to yield satisfactory results. As the height of the structure increases a significant upward leader activity is expected and therefore the model employed must consider that. The works by Eriksson [6], Dellera el al. [7] and Rizk [8] have mainly considered the upward leader activities during the attachment process. However, due to the associated complexity, only the macroscopic models, which are originally developed for modelling long-air-gap breakdown under switching impulse, are adopted in their work. These three works are discussed in detail for three important aspects.
Field intensification: For the calculation of electric field, all three works have employed numerical field calculation using the well-known charge simulation method (CSM).

Upward leader inception: For deciding the instant of upward leader inception, Eriksson [6] and Dellera et al. [7] employed the concept of 'critical size'. Accordingly, the instant of leader inception is the same as the instant of corona inception on a $35 \mathrm{~cm}$ radii sphere situated at the top of the tower and a $10 \mathrm{~cm}$ radius cylinder placed concentric to the horizontal conductors/ground wires. In the model by Rizk, a height-dependent critical radius has been employed, deduced in his earlier work.

Upward leaderpropagation and finaljump: In the work of Eriksson an equal velocity ratio has been considered for both upward and descending leaders. However, the method describing both propagation and final jump is more of a geometric nature and therefore is not considered further.

In the work of Dellera et al. the velocity of propagation of the descending leader and that of the upward leader is initially taken to be in the ratio $4: 1$, later reduced to $1: 1$. They envisaged a streamer ahead of the leader the extent of which is determined by an average gradient of $500 \mathrm{kV} / \mathrm{m}$ along the maximum field strength line. The linear charge density on the latest length of the descending leader as well as that on the upward leader is assumed to be 50 and $100 \mu \mathrm{C} / \mathrm{m}$, respectively, for the positive and negative leaders. These values were deduced from the laboratory breakdown experiments on long air gaps. The remaining portion of the descending leader is assigned a charge density given by $\rho=38 I^{0.88}$, where $p$ is in $\mu \mathrm{C} / \mathrm{m}$ and current $I$ is in $\mathrm{kA}$. Cloud charge was also modelled by an equivalent unipolar charge. They applied their method to analyse shielding failure in transmission lines for different orographic conditions. The two values for the velocity ratio and their instant of changeover as demanded by Dellara et al.'s model is rather difficult to comprehend and apply. Further, the effect of cloud charges seems to be significant only for tall structures and for upward flashes, which is not expected for the case under study. For these reasons their model was not adopted for the present work.

Rizk also has assumed equal velocity ratios for the upward and downward leaders. Similar to Eriksson, Rizk has assumed the charge density on the descending leader to be linearly varying with a maximum occurring at the tip and reducing to zero at the root. The total charge on the leader was obtained using

$$
Q=0.076 I^{0.68} \quad(\mathrm{C}, k A)
$$

Considering the upward leader as an electric arc, he deduced an expression for its axial voltage gradient. A first-order model for the arc, along with the velocity of propagation of upward leaders [8], was employed for this purpose. Then, instead of fixing the charge on the upward leader, its voltage gradient was fixed by the simplified expression given in [8]

$$
\Delta U_{l}=-(3 l+73.393) \quad(k V)
$$

where, $\mathrm{I}$ is the length of the leader and $\Delta U_{l}$ is the voltage difference across its ends. The instant of final jump is governed by the existence of an average gradient of $500 \mathrm{kV} /$ $\mathrm{m}$, which is the net gradient of positive and negative streamers bridging the gap. Rizk has employed his model to analyse the attractive radii and the probability of strike to tall standing structures.

The model by Rizk seems to be more versatile in simulating the physical process. Due to the resemblance between the leader and the electric arc it would be more appropriate to fix the gradient along it rather than the 
charge. The local electric field would naturally fix the required charge along it. Further, there is no change in the velocity of two leaders and therefore it is relatively simple to apply. Good correlation has been shown between the field data of lightning incidence and that predicted by the model for tall isolated towers. Considering all these, the basic model proposed by Rizk was considered for the present work.

In all three models, if the height of the descending leader tip reduces below that of the upward leader tip (without any encounter), a ground strike is assumed to occur.

\section{Present work}

Earlier works were mostly concerned with either power transmission lines or isolated tower/conductors. The present problem differs from them mainly in two items. Due to the difference in geometry the net electric field intensification governing the instant of upward leader inception is very different. Further, in the present problem, there are multiple prospective points of upward leader inception. Therefore some modifications become necessary to the Rizk model to incorporate these, discussed as follows.

The electric field at the tip of the structures under the influence of a descending leader has to be determined accurately. The present work, like the earlier works, employs the charge simulation method for this purpose. All the structures are modelled using point charges and finite line charges, and the potential error was kept below $1 \%$ at all places [2]. The necessary details are provided in the Appendix (Section 8.1).

\section{I Modified criteria for upward leader inception}

The inception of the upward streamers/leaders is governed by the local electric field at the top of the structure. Rizk [8] has restated this condition in terms of the ambient potential (induced by the descending leader) for both isolated tower and conductor. Rizk employs a height-dependent critical radius for both types of geometries. According to him the ambient potential $U_{i}$ (i.e. potential in the absence of the structure) required for the inception of the upward leader [8] is given by

- for a shield wire of radius $a$ and height $h$

$$
U_{i}=\frac{2247}{1+\frac{5.15-5.49 \ln a}{h \operatorname{In} \frac{2 h}{a}}}
$$

where both $\boldsymbol{a}$ and $\boldsymbol{h}$ are in metres, and

- for a tower of height $h$ (in metres)

$$
U_{i}=\frac{1556}{1+\frac{3.89}{h}}(k V)
$$

However, in the present problem the presence of other nearby earthed structures significantly reduces the electric field induced by the charges on the descending leader. The electric field on the shield wire in the system is less by a factor of 2.39 when compared with the field on the same shield wire kept in isolation. Similarly, the field on the tower in the system is less by a factor of 1.39 , as compared with that on the same tower considered in isolation. The presence of LPS reduces the field on LV by a factor of 1.65 . Therefore Rizk's criteria cannot be applied directly.

Incidentally, numerical field calculations for the geometry of the problem considered have revealed the following. Due to a large separation between the neighbouring structures the local field flux line pattern at the top of structures (extending up to few decimetres) remains practically unaffected, and only their magnitude gets reduced. Considering this and noting that the local electric field is responsible for the inception of upward leaders, it is more appropriate to restate Rizk's criteria in terms of local electric gradients. To obtain them, numerical simulations were carried out. The main leader was made to descend vertically along the mid-span position of an isolated shield wire and also along the axis of an isolated tower until the corresponding continuous leader inception potentials given by (5) and (6) are reached. The electric field on the surface of the structure at this instant is computed. These fields, which serve as the modified inception criteria, are evaluated to be $3.7 \times 10^{7}$ and $3.0 \times 10^{6} \mathrm{~V} / \mathrm{m}$ for the shield wires and towers, respectively. In the calculation, for an accurate evaluation of the electric field profile on the tower top, a sphere of critical radii $(33 \mathrm{~cm}$ for a height of $130 \mathrm{~m}$ as deduced from Rizk's work) was employed. As the equivalent tip radii of the UT and LV are greater than the critical radii, the inception is said to occur when the field on the hemispheres of equivalent radii placed at their top reach a value of $3 \times 10^{6} \mathrm{~V} / \mathrm{m}$.

To assess the ability of the individual members on their upward leader launching ability, a main leader was made to descent at three different places viz. exactly on the vertical line through the axis of the tower, mid-span of one of the shield wires and through the axis of the LV. The ambient ground field at the instant of upward leader inception are found to be $1.53 \times 10^{4}, 4.44 \times 10^{4}$ and $1.47 \times 10^{5} \mathrm{~V} / \mathrm{m}$ for the tower, shield wire and LV, respectively. It is clear from this that the tower has the highest ability to launch connecting leaders.

\subsection{Modified propagation criteria}

Both Eriksson and Rizk have taken the velocity ratio of descending and upward leaders to be unity. However, in the system under study, the competition amongst the multiple upward leaders can reduce their tip fields, thus hampering their progress. In the absence of credible data on the upward leader propagation characteristics, the incremental length of the upward leaders are calculated as follows. For an arbitrarily small length of the upward leaders, 41 (as computed using the equal velocity ratio) in the direction of the line connecting the tips of the upward leader and the descending leader, existence of the (positive) streamer propagation gradient of $400 \mathrm{kV} / \mathrm{m}$ [8] is first checked. If it is not satisfied, $\Delta l$ is reduced in a fixed length $(0.75 \mathrm{Al}, 0.5 \mathrm{Al}$ and 0.2541 ) until the condition is satisfied. A fixed radius for the upward leader charge envelope is assumed $(10 \mathrm{~cm}$ in the present case) and the charge on it is numerically computed using the leader voltage drop (4).

With these modifications to the Rizk model, simulation of the attachment process is carried out mainly for negative lightning. The main aim of these simulations is to determine the maximum magnitude of bypass current, distribution of bypass current within the area projected by LPS, probability of a bypass stroke (and hence the flash), the attractive radii of LPS and the average number of negative flash to LPS in a year.

\section{Simulation results}

Earlier works [6-8] have considered a significant branch of the leader descending vertically along a straight line. To account for the randomness of the lightning path, the present work has also made a study with inclined leaders. 
The simulation results indicate that the vertical descent forms the worst case and therefore was employed in for all the analyses.

\subsection{Maximum bypass current}

During the descent of the stepped leader, the ambient ground field increases. This increase in field is dependent on the charge contained in the leader, which is related to the prospective return stroke current peak. The higher the current, the greater is the ambient field. Therefore 1 he field at the top of the structure is dependent on the prospective return stroke current. Any conventional lightning protection system by virtue of their geometry possesses higher field intensification at the top, which enables them to initiate an upward leader/streamer well before that from the system to be protected. However, in the protection systems, which are isolated from the system being protected due 1.o their physical separation, upward leaders from the protection system may not always be able to intercept the descending leader. Such a shielding failure is encountered with the lowcurrent strokes descend close to the system to be protected. For the present case, both the maximum magnitude of the current in such a bypass stroke as well its probability are important.

As can be expected, the vertical stroke descending directly on to the launch vehicle is the bypass stroke of highest magnitude. For the problem under study its value is found to be $14 \mathrm{kA}$. For strokes with higher currents interception is ensured. Fig. 2 shows the simulation results for a $15 \mathrm{kA}$



Fig. 2 Simulation results showing successful stroke interceptionfor IS $k A$ negative stroke

$C$ - Connecting leaders from masts on tower

$\mathbf{J}$ - Final jump distance (shown for only one connecting leader)

stroke. The final jump distance (where a streamer bridging takes place between the descending and upward leaders) is also marked for one of the leaders in the Figure.

During the simulations all points attaining the leader inception field have been assumed to have equal ability to launch an upward leader. The direct application of the Rizk's equation (which were developed for isolated tower and conductors) yields the maximum bypass stroke to be $6 \mathrm{kA}$, and predicts an interception by the shield wires for slightly higher currents. However, in the present case, due to the reduction in field on the shield wires, they are found to be incapable of doing so. Only for leaders descending close to shield wires is interception by the shield wires possible. A detailed description of the simulation results can tie found in [2].

The main intention of placing six shield wires is to effectively distribute the stroke currents to other towers and thereby limit the potential rise at the struck point.

During interception of the strokes descending vertically above the area confined by the LPS, many upward leaders are found to be equally competitive in establishing contact. The mutual interaction of their fields at this stage can hamper their further progress and attachment. However, simulations for the bypass strokes showed that the associated large final jump distances have caused an insignificant interaction. For strokes with larger currents, interception by towers is ensured and hence the mutual interaction effects of connecting leaders become unimportant.

\subsection{Probability of bypass strokes}

The maximum bypass current computed in the previous Section is itself incomplete unless the associated probabilities are also given. This can be computed if the spatial distribution of bypass currents, the cumulative probability of lightning currents, and the local ground flash density are known. The spatial distribution of the bypass current magnitudes is obtained through simulation. To minimise the computation involved the maximum bypass currents were computed along some salient directions and interpolated to other places. The lightning current has a lognormal distribution [9] with a median current of $31 \mathrm{kA}$. $P(I)$, the probability of occurrence of a stroke having a magnitude $\geq I$, where $I$ is in $\mathrm{kA}$ can be approximated by [9]

$$
P(I)=\frac{1}{1+\left(\frac{I}{31}\right)^{2.6}}
$$

Therefore cumulative probability of a stroke with magnitude less than $I$ is $F(I)=1-P(I)$. Further, the occurrence of lightning flashes in any given area can be computed using the isokeraunic level $(T d)$, which is reported to be in the range of 50-90 thunderstorm-days per year for the location of the Indian satellite launch pads. Then the average ground flash density $N_{g}$ is given by [4]

$$
\begin{aligned}
N_{g} & =k(T d)^{\alpha}=0.023(T d)^{1.3} \\
& =[3.7-8] \text { flashes per } \mathrm{km}^{2} \text { per year }
\end{aligned}
$$

As the probability of a lightning flash in a given area and the probability of a stroke with specified peak current can be considered as independent of each other the combined probability is computed as their product.

The overall probability of the bypass flash is found to be one flash per 1095-510 years for the value of $T d$ between $50-90$ thunderstorm-days per year. To further demonstrate the efficacy of the protection system the maximum of the value is normalised with the average flashes per year corresponding to the area occupied by LPS, and the resulting value is found to be $1.75 \%$.

\subsection{Attractive area of LPS}

A knowledge of the attractive area projected by the LPS would enable us to ascertain the protection offered by the LPS for small structures in the vicinity (structures outside the LPS). Such a study would also give an idea of percentage of extra flashes, which are invited by the LPS to itself. However, these will be dependent on the prospective return stroke currents associated with the strokes.

The computed attractive area is shown in Fig. 3. Due to a coarse set of points employed for the computation, sharp edges are seen on the Figure. A very accurate locus was not needed and hence not attempted.

For the computation of the probability of flashes per year within the area projected by the LPS, the attractive area projected by the LPS has been interpolated from the simulation results. Using the ground flash density data and 


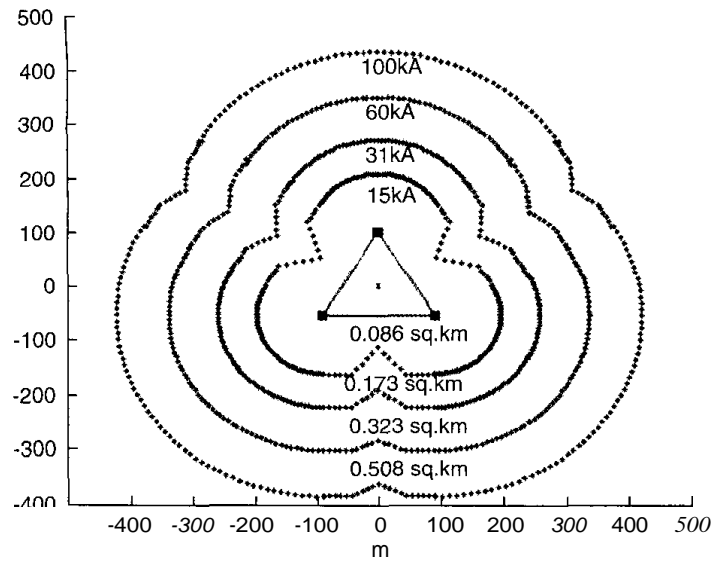

Fig. 3 Loci of attractive area projected by lightning protection system

cumulative probability distribution of the lightning currents, the numbers of flashes per year is estimated. The attractive area is found to increase almost linearly with the peak magnitude of the stroke current. Fig. 4 gives the probability

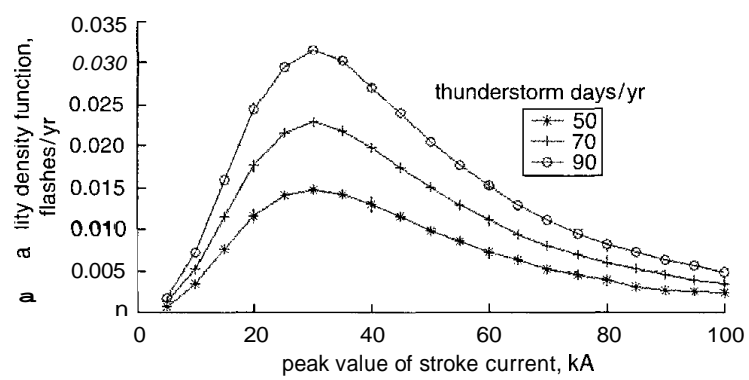

Fig. 4 Probability density function of number of flashesper year to protection system us function oj peak stroke current

density function of the number of flashes to the protection system as a function of the peak current. As with any lightning protection system, while offering protection against direct strokes, LPS is inviting strokes onto itself, thus increasing the actual lightning activity in the launch pad area. The number of strokes to LPS has been estimated to be less than 2 flashes per year.

\section{Some additional analysis}

All the preceding analysis was carried out for a fixed height of the protection system, a fixed leader root height, and negative descending leaders. For ascertaining the influence of these parameters on the protective action some additional simulations were carried out.

To check the optimality of the height of the LPS, simulations were carried out for the bypass strokes with height of the LPS as the variable. Results show that the maximum current in the bypass stroke vanes very slowly at a rate of $0.1 \mathrm{kA} / \mathrm{m}$ with the height of the LPS (around the present height). Therefore it is uneconomical to increase the height of the LPS further.

In all of the simulations, following the work of Eriksson [6] and Rizk [8] a significant branch of the descending leader with a root height of $2.5 \mathrm{~km}$ only was considered. Considering the randomness of the lightning phenomena, an analysis was taken up for the maximum current in a bypass stroke with leader root height as a variable. To keep the magnitude of the prospective return-stroke current approximately the same, the maximum linear charge density appearing at the leader tip was maintained the same. These simulation results show that the maximum current in the bypass stroke varies at a rate of $3 \mathrm{kA} / \mathrm{km}$ with the leader root height (varied around $2.5 \mathrm{~km}$ ).

All this analysis was for a negative lightning which represents $>90 \%$ of the flashes in most of the places. The positive strokes seem to have gained less attention in the literature than the negative ones. However, for the sake of completeness, an attempt was made in the present work to make a rough estimate of maximum bypass current under positive lightning. By assuming both the leader inception and the propagation gradients for the negative upward leaders to be approximately twice that of positive [10], the maximum bypass current was determined to be $38 \mathrm{kA}$.

A similar analysis has been carried out for the launch pad-II, which is currently under execution. The results obtained for pad-II have been presented in [11].

\section{Summary}

The lightning protection system to the Indian satellite launch pad has been investigated for its stroke interception efficacy. Basically, the air termination network of the lightning protection system is responsible for stroke interception. For an accurate evaluation of its interception efficacy, a model with abilities to represent the different stages associated with air breakdown process is necessary. Such a model has been proposed in the literature, which is applicable for isolated structures. The present work has attempted some modifications to it so as to account for the interaction of various elements of protection system, as well as the system under protection, and the inception and propagation of multiple upward leaders. With the modified method a more reliable evaluation of the efficacy of the air termination system of the Indian satellite launch pad lightning protection scheme has been performed for negative flashes. The simulation results show that the maximum current in a bypass stroke is $14 \mathrm{kA}$ and the probability of a bypass flash is one flash per 1095-510 years (for $T d$ between 5090 thunderstorm-days per year). Further, the attractive area of the protection system has been computed along with probability of a flash to LPS. The number of flashes to LPS has been estimated to be less than two flashes a year.

In addition, some sensitivity analysis was also carried out to determine the dependency of bypass stroke on height of the protection system and total length of the descending leader. Lastly, an attempt was also made to arrive at a rough estimate of maximum bypass current under positive lightning.

The methodology developed and employed in the present work is quite general and hence is applicable to any passive lightning protection scheme including the insulated mast design. The significance of the methodology becomes evident in systems wherein the electric field interaction amongst the protection and protected system are significant and there are multiple prospective points of inception.

\section{Acknowledgment}

The authors acknowledge the valuable suggestions made by the referees in improving the presentation of the paper. 


\section{References}

1 KUMAR, U., and NAGABHUSHANA, G.R.: 'Analysis of lightning protection system for indian satellite launch pad'. National Interagency Coordination Group Lightning Conference, Orlands, Florida, April 2000

2 JOSEPH, N.T.: "Analysis of the protective action of a closely distributed lightning protection system for a satellite launch pad'. Masters Thesis, Department of High Voltage Engineering, Indian Institute of Science, 2000

3 VAN BRUNT, R.J., NELSON, T.L., and STRICKLETT, K.L.: 'Early streamer emission lightning protection systems: An overview', IEEE DEIS Mag., 2000, 16, (1), pp. 5-24

4 'IEEE Working Group Report.: 'Estimating lightning performance of transmission lines-11: Updates to analytical model', IEEE Trans. Power Deliv., 1993, 8, (3), pp. 1254-1267

5 HORVATH, T.: 'Computation of lightning protection', (Wiley, 1991)

6 ERIKSSON, A.J.: 'The incidence of lightning strikes to power lines', IEEE Truns. Power Deliv., 1987, 2, (3), pp. 859-870

7 DELLERA, L., and GARBAGNATI, E.: 'Lightning stroke simulation by means of the leader progression model, Part I: Description of the model and evaluation of freestanding structures', IEEE Truns. the model and evaluation of freestandi

8 RIZK, F.: 'Modeling of lightning incidence to tall structures, Part I \& II', IEEE Truns. Power Deliv., 1994, 9, (1), pp. 162-193

9 IEEE Working Group on Estimating Lightning Performance of Transmission Lines.: 'A simplified method for estimating lightning performance of transmission lines', IEEE Truns. Power Appar. Syst, 1985, 104, (4), pp. 919-932

10 RIZK, F.: 'Critical switching impulse strength of phase-to-phase air insulation', IEEE Trans. Power Deliv., 1993, 8, (3), pp. $1492-1506$

11 JOSEPH, N.T., and KUMAR, U.: 'Evaluation of the protective action of LPS to Indian satellite launch Pad-II'. Paper 2.2., 12th International Symposium on High Voltage Engineering, 2001 Bangalore

12 MALIK, N.H. 'A review of the charge simulation method and its applications', IEEE Truns. Electr. Instl., 1989, 24, (1), pp. $3-20$

\section{Appendix}

\subsection{Charge simulation method}

In this method the actual electric field distribution is simulated by employing a set of discrete charges, which are placed outside the region of interest [12]. By enforcing the applied and/or natural boundary conditions at some suitably selected points on the boundaries called contour points, an inhomogeneous set of linearly independent equations can be obtained in terms of the unknown charge magnitudes. On solving these equations, unknown charge magnitudes can be obtained.

\subsection{Modelling of structures}

The lightning protection system (LPS), umbilical tower (UT) and launch vehicle (LV) are modelled using uniform line charge segments and point charges. Suitable modelling scheme has been adopted for minimising the computation without sacrificing the accuracy of the computed field on the aerial receptor system. Modelling details follow:

8.2. I Towers: Each tower is $120 \mathrm{~m}$ tall and has square cross-section. Towers have a tapering cross-section up to a height of $100 \mathrm{~m}$ and remain uniform thereafter. The four main poles of the tower are interconnected by many horizontal and diagonal lattice elements. These are found to have a negligible influence on the electric field at the aerial receptor system and hence are not modelled. For the tower legs, contour points are placed on their surface and the corresponding line charges are placed inside (refer to Fig. 5). Detailed modelling of the legs is not required, as the field on it is not important. From $100 \mathrm{~m}$ onwards, tower has uniform cross section of $2.3 \times 2.3 \mathrm{~m}$. For its modelling, horizontal line charges are placed on all the four faces. The corresponding contour points are placed at middle of the

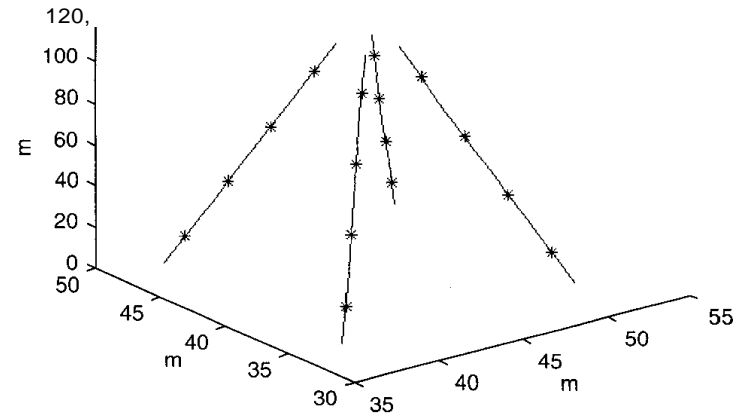

Fig. 5 Schematic showing charges and contour points on tower legs

(*indicates location of contour points)

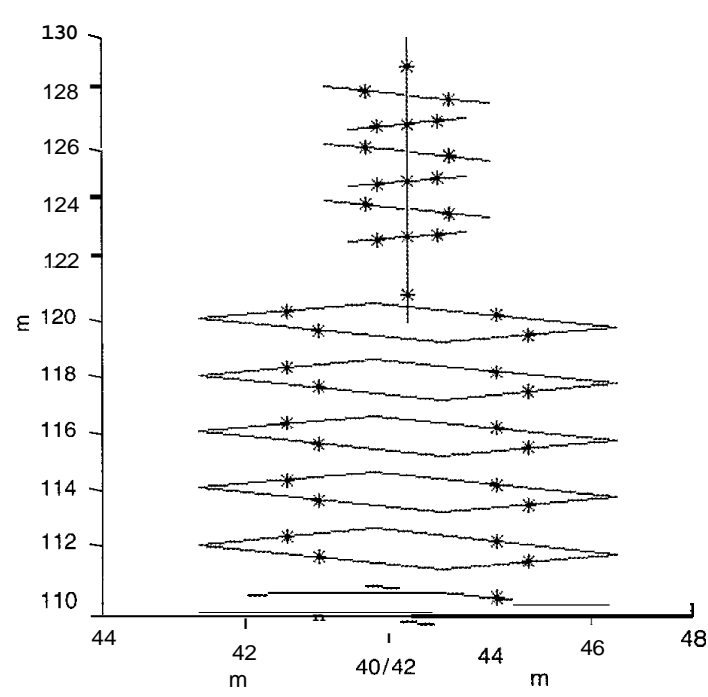

Fig. 6 Schematic showing charges and contour points at tower top (*indicates location of contour points)

faces as shown in Fig. 6. At the top of the tower a $10 \mathrm{~m}$ tall mast $(10 \mathrm{~cm}$ diameter $)$ stands vertically to which 12 horizontal cross-arms of $2 \mathrm{~cm}$ diameter each are arranged. The vertical gap spacing between them is $2 \mathrm{~m}$ and their length is $1.1 \mathrm{~m}$. As shown in Fig. 6, line charges are placed at the centre of these cylindrical members and corresponding contour points are placed on their surface.

8.2.2 Shield wires: A set of six shield wires is arranged in three rows with a horizontal spacing of $2 \mathrm{~m}$ and a vertical spacing of $5 \mathrm{~m}$. The top-most set is hung at a height of $120 \mathrm{~m}$ on the tower. The shield wires have sag of $8 \mathrm{~m}$. They are modelled as cylindrical conductors with a radius of $6 \mathrm{~mm}$. Line charge segments are employed for modelling of the shield wires. Charges are kept at the centre of shield wires and the corresponding contour points are placed on the surface, as shown in Fig. 7).

8.2.3 Launch vehicle: The LV is approximately a cylindrical structure with a radius of $1.45 \mathrm{~m}$ and a height of $55 \mathrm{~m}$. Line charge segments are employed for modelling of 


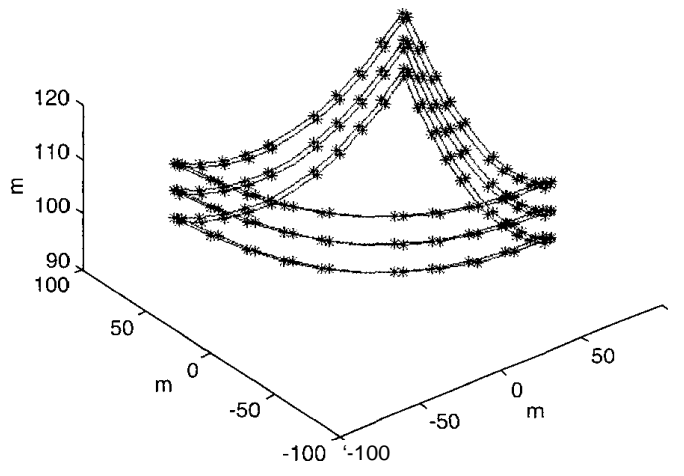

Fig. 7 Schematic showing charges and contour points on shield wives

(*indicates location of contour points)

the LV. The tip of the LV is modelled by using line and point charges.

8.2.4 Umbilical tower: UT is separated by a distance of $5.45 \mathrm{~m}$ from LV. The height of the UT is $51 \mathrm{~m}$ and it is cubical in shape with a cross-section of $3 \times 3 \mathrm{~m}$. Line charge segments are employed for its modelling. For calculating the leader inception field from UT, the concept of equivalent radius is employed.

\subsection{Equations employed}

As point charges and line charges are used in the system for simulation, to compute the potential at any point in $3 \mathrm{D}$ space the following equations are used.

- Potential at any point $(x, y, z)$ in air due to a uniform line charge with image charge on earth

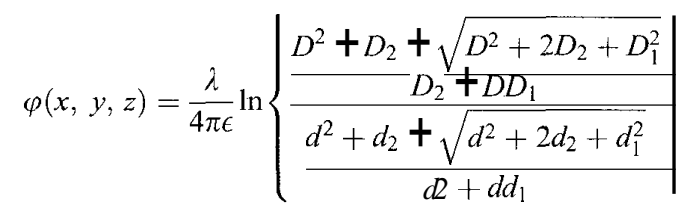

where,

$$
\begin{aligned}
d= & D=\sqrt{\left(x_{2}-x_{1}\right)^{2}+\left(y 2-y_{1}\right)^{2}+\left(z_{2}-z_{1}\right)^{2}} \\
D_{1}= & \sqrt{\left(x-x_{1}\right)^{2}+\left(y-y_{1}\right)^{2}+\left(z-z_{1}\right)^{2}} \\
D_{2}= & \left(x_{2}-x_{1}\right)\left(x_{1}-x\right)+\left(y_{2}-y_{1}\right) \\
& \times\left(y_{1}-y\right)+\left(z_{2}-z_{1}\right)\left(z_{1}-z\right) \\
d_{1}= & \sqrt{\left(x-x_{2}\right)^{2}+\left(y-y_{2}\right)^{2}+\left(z+z_{2}\right)^{2}} \\
d_{2}= & \left(x_{1}-x_{2}\right)\left(x_{2}-\mathbf{x}\right)+\left(y_{1}-y 2\right) \\
& \times(Y 2-y)+\left(z_{1}-z_{2}\right)\left(-z_{2}-z\right)
\end{aligned}
$$

where $x_{1}, y_{1}, z_{1}$ and $x_{2}, y_{2}, z_{2}$ are the co-ordinates of starting and ending points of the line.
- Similarly, due to a point charge

$$
\begin{aligned}
& \varphi(x, y, z)=\frac{Q}{4 \pi \epsilon}\left\{\frac{1}{D_{3}}-\frac{1}{d_{3}}\right\} \\
& D_{3}=\sqrt{\left(x-x_{3}\right)^{2}+\left(y-y_{3}\right)^{2}+\left(z-z_{3}\right)^{2}} \\
& d_{3}=\sqrt{\left(x-x_{3}\right)^{2}+\left(y-y_{3}\right)^{2}+\left(z+z_{3}\right)^{2}}
\end{aligned}
$$

where $\left(x_{3}, y_{3}, z_{3}\right)$ is the location of point charge in air.

- The fields in any direction ' $n$ ' can be obtained by using $E_{n}=\partial \phi / \partial n$. In the numerical field computation this has been approximated by the finite difference method.

- For an accurate evaluation of the field on the shield wires, the self-field component is analytically evaluated.

In accordance with the earlier works, the stepped leader is assumed to descend from the cloud at a height of $2.5 \mathrm{~km}$. The charge in the leader is maximum at its tip (bottom), which reduces linearly to zero at its root (top). The charge density $\lambda$ can be computed from the expression that relates the total charge in the leader to the peak of the prospective return stroke current $[6,8]$ :

$$
Q=0.076(I)^{0.68}
$$

The equations for evaluating the potential at any point due to leader are as follows.

- Potential along the axis of the leader length $l_{\mathrm{z}}$ descending vertically

$$
\begin{aligned}
\varphi(z)= \\
\\
\left.\qquad \begin{array}{l}
\sqrt{R^{2}+\left(l_{z}+y\right)^{2}}-\sqrt{R^{2}+y^{2}}- \\
\sqrt{R^{2}+(2(d-\mathrm{i}=)+I,-y)^{*}} \\
+\sqrt{R^{2}+(2(d-l,)-y)^{2}-} \\
\left(y+l_{z}\right) \ln \left[\frac{\sqrt{R^{2}+\left(l_{z}+\cdots\right)^{2}}+I,+y}{\sqrt{R^{2}+y^{2}}+y}\right] \\
+\left(2\left(d-l_{z}\right)+l_{z}-y\right) \\
\ln \left[\frac{\sqrt{R^{2}+\left(2\left(d-l_{z}\right)+l_{z}-y\right)^{2}}+2\left(d-l_{z}\right)+l_{z}-y}{\sqrt{R^{2}+\left(2\left(d-l_{z}\right)-y\right)^{2}}+2\left(d-l_{z}\right)-y}\right]
\end{array}\right]
\end{aligned}
$$

where $R$ is the radius of the channel, $y$ is the distance of the measurement point from the leader tip along the axis and $d$ is the total length of the leader. The radius of the channel is computed by

$$
R=\frac{\lambda_{1}}{2 \pi \epsilon E}
$$

where $E$ is the critical corona field, the value being $3 \times 10^{6} \mathrm{~V} / \mathrm{m}$ at standard atmospheric pressure and temperature.

- Potential at a horizontal distance $r$ from the leader descending vertically. Considering the leader as a line charge 
descending vertically

$$
\begin{aligned}
& \varphi(r, z)= \\
& \frac{\lambda_{1}}{4 \pi \epsilon} \ln \begin{array}{l}
\sqrt{r^{2}+\left(l_{z}+p\right)^{2}}-\sqrt{r^{2}+p^{2}}- \\
\sqrt{r^{2}+q^{2}}+\sqrt{r^{2}+\left(l_{z}+q\right)^{2}}+\left(p+l_{z}\right) \\
\ln \left[\frac{\sqrt{r^{2}+\left(l_{z}+p\right)^{2}}+p+l_{z}}{\sqrt{r^{2}+p^{2}}+p}\right]+\left(q+l_{z}\right) \\
\operatorname{In}\left[\frac{\sqrt{r^{2}+\left(l_{z}+q\right)^{2}}+q+l_{z}}{\sqrt{r^{2}+q^{2}}+q}\right]
\end{array} \mid
\end{aligned}
$$

where $p=d-l_{z}-z$ and $q=d-l_{z}+z$

In the case of computation of leader tip potential, the total leader length is split into a number of equal segments. Each segment has its own radius, depending on its charge. The potential at the tip of the leader is the sum of the potentials due to each leader segment. If $z$ is measured along the leader axis and $z_{l}, z_{2}$ are the co-ordinates and $\lambda_{1}$ and $\lambda_{2}$ are the linear charge densities of the starting and ending of the segment, then the potential at $z$ is given by

$$
\begin{aligned}
& \varphi(z)=\left(\frac{1}{2 \pi \varepsilon R^{2}}\right)[ \\
& \frac{\lambda_{1}}{\left(z_{2}-z_{1}\right)}\left\{\begin{array}{l}
\frac{\left(z-z_{2}\right)^{2}}{2} \sqrt{R^{2}+\left(z-z_{2}\right)^{2}}-\frac{\left(z-z_{1}\right)\left(z-z_{2}\right)}{2} \\
\quad \times \sqrt{R^{2}+\left(z-z_{1}\right)^{2}}-\frac{\left(z_{2}-z\right)}{2} \\
R^{2} \ln \left(\frac{z-z_{2}+\sqrt{R^{2}+\left(z-z_{2}\right)^{2}}}{z-z_{1}+\sqrt{R^{2}+\left(z-z_{1}\right)^{2}}}\right) \\
-\frac{\left(z-z_{2}\right)^{3}}{2}-\frac{\left(z_{2}-z\right)\left(z-z_{1}\right)^{2}}{2} \\
-\frac{\sqrt[3]{R^{2}+\left(z-z_{2}\right)^{2}}}{3} \\
+\frac{\sqrt[3]{R^{2}+\left(z-z_{1}\right)^{2}}}{3}+\frac{\left(z-z_{2}\right)^{3}}{3}-\frac{\left(z-z_{1}\right)^{3}}{3}
\end{array}\right. \\
& +\frac{\lambda_{2}}{\left(z_{2}-z_{1}\right)}\left\{\begin{array}{l}
\frac{\left(z-z_{1}\right)^{2}}{2} \sqrt{R^{2}+\left(z-z_{1}\right)^{2}} r \\
-\frac{\left(z-z_{1}\right)\left(z-z_{2}\right)}{2} \\
\times \sqrt{R^{2}+\left(z-z_{2}\right)^{2}}-\frac{\left(z-z_{1}\right)}{2} \\
R^{2} \ln \left(\frac{z-z_{2}+\sqrt{R^{2}+\left(z-z_{2}\right)^{2}}}{z-z_{1}+\sqrt{R^{2}+\left(z-z_{1}\right)^{2}}}\right) \\
\left(z-z_{1}\right)^{3} \frac{\left(z_{1}-z\right)\left(z-z_{2}\right)^{2} .}{2} \\
+\frac{\sqrt[3]{R^{2}+\left(z-z_{2}\right)^{2}}}{3} \\
-\frac{\sqrt[3]{R^{2}+\left(z-z_{1}\right)^{2}}}{3}-\frac{\left(z-z_{2}\right)^{3}}{3}+\frac{\left(z-z_{1}\right)^{3}}{3}
\end{array} \mid\right.
\end{aligned}
$$

$$
\begin{aligned}
& \varphi(z)=\left(\frac{1}{2 \pi \varepsilon R^{2}}\right) !
\end{aligned}
$$

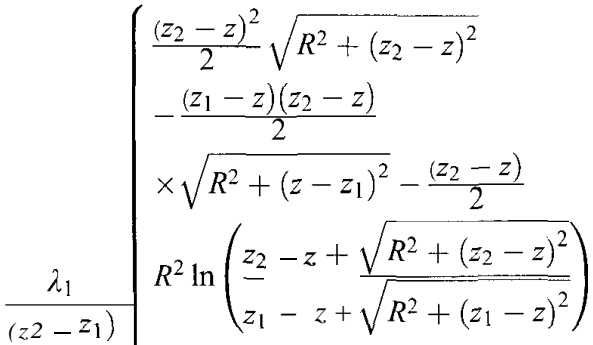

$$
\begin{aligned}
& \left(z_{2}-z\right)^{3} \quad \frac{\left(z_{2}-z\right)\left(z-z_{1}\right)^{2}}{2} \\
& \sqrt[3]{R^{2}+\left(z_{2}-z\right)^{2}} \\
& \frac{\sqrt[3]{R^{2}+\left(z_{1}-z\right)^{2}}}{3}+\frac{\left(z_{z} z\right)^{3}}{3}-\frac{\left(z_{1} z\right)^{3}}{3} \mid \\
& \left\{\begin{array}{l}
\frac{\left(z_{1}-z\right)^{2}}{2} \sqrt{R^{2}+\left(z_{1}-z\right)^{2}} \\
-\frac{\left(z-z_{1}\right)\left(z_{2}-z\right)}{2} \\
\sqrt{R^{2}+\left(z_{2}-z\right)^{2}}-\frac{\left(z-z_{1}\right)}{2}
\end{array}\right. \\
& +\frac{\lambda_{2}}{\left(z 2-z_{1}\right)}\left\{R^{2} \ln \left(\frac{z_{2}-z+\sqrt{R^{2}+\left(z_{2}-z\right)^{2}}}{z_{1}-z+\sqrt{R^{2}+\left(z_{1}-z\right)^{2}}}\right)\right. \\
& -\frac{\left(z_{1}-z\right)^{3}}{2}-\frac{\left(z-z_{1}\right)\left(z_{2}-z\right)^{2}}{2} \\
& \begin{array}{l}
+\frac{\sqrt[3]{R^{2}+\left(z_{2}-z\right)^{2}}}{3}- \\
\frac{\sqrt[3]{R^{2}+\left(z_{1}-z\right)^{2}}}{3}-\frac{\left(z_{2}-z\right)^{3}}{3}+\frac{\left(z_{1}-z\right)^{3}}{3}
\end{array} \\
& 1 \\
& z<=z_{1} \ldots
\end{aligned}
$$

\subsection{Error in computed potential}

To have good accuracy in the computed potential the distances between contour points and charges have to be controlled. The ratio of the distances between successive contour points and that between the contour point and the corresponding charge is termed as assignment factor [12]. Literature suggests that, for a good accuracy, assignment factor must lie between 1.0-2.0, and the same has been employed in the present work. In CSM, the potential error is normally checked at the points between two contour points called the checkpoints. At these check points the percentage error was calculated as.

$$
\% \text { error }=\left(\frac{\varphi-\varphi^{\prime}}{\varphi}\right) 100
$$

where $\varphi$ is the potential at the checkpoint due to the leader and $\varphi^{\prime}$ is the potential at the same checkpoint by the induced charges on the structures. The potential error was found to be within $0.01 \%$ to $1 \%$ in any part of the system. 